\title{
Public Awareness of Science
}

An address by M. Brock Fenton, Department of Biology, York University

In Canada, the lack of a strong public awareness or appreciation of science is often blamed on the media, but, in reality, scientists have contributed to it. Saying this does not demean or undervalue the efforts that some scientists have made to increase public awareness of science because, for the most part, Canadian scientists have no tradition of communicating outside their disciplines.

\section{General Problems}

Some of the hurdles to increasing public awareness are common to science in general. Six come to mind:

1. The way people experience science in school. Too often it is presented as a litany of facts that must be memorized and accurately regurgitated. This approach to science education often is justified by the notion that some facts must be learned to establish a foundation for the next level. To practicing scientists, however, science is the process of finding answers, not memorizing them. Furthermore, the thrill of science is finding the answers, and the quest for knowledge is what captivates most scientists. When a sense of the unknown is missing from science education, students will equate science with the simple accumulation of factual knowledge. 
2. Society's expectation that science will provide the "right" answers to questions and solutions.to our problems, particularly, for example, those related to the environment or health. When science is perceived as the source of truth, people are dismayed to see scientists expressing different interpretations of the same evidence. If science were thought to represent a process (the first problem), then this conflict could be alleviated.

3. Many scientists are not strong communicators. I suspect that some people enter science to minimize their interactions with other people. By using jargon and selecting intimidating words, scientists can appear knowledgeable and aloof. By being picky and critical, scientists can isolate themselves from all but the group who understands what they say and are not alienated by it. To increase public awareness of science, scientists must change their demeanour.

4. Too many scientists working in "pure" or basic science have little respect for their colleagues whose research is applied. This dichotomy, often accentuated by the structure of university departments or research institutes, erects barriers to communication within science. This attitude often means that some scientists avoid exploring the more practical aspects of their work.

5. By describing their basic research as "curiousity-driven" or "hobby" work, scientists convey the impression that it is a luxury and an indulgence. At times when there is less and less money available for research and increasing pressure for scientists to do applied work, it is easy for the public and, in turn, for those who establish policy to reduce support for basic research. The combination of the description of the work and the pressure of limited resources makes it easy to overlook the countless examples of basic work that has produced long-term applied benefits.

6. Too often scientists give little credit to their colleagues who work to increase public awareness of science. This lack of appreciation often extends to active resentment. David Suzuki is an example of someone whose effort to increase public awareness of science have brought him wide respect in the public's eyes and considerable hostility from some parts of the scientific community.

I think that scientists must recognize and address these six problems. An alternate route, namely making complaining about a lack of support as their only public awareness of science activity, is unlikely to change the situation. Complaining makes science appear as yet another item requiring support from the public purse. Most taxpayers and politicians will not see science to be as urgent or deserving as matters of health and other social services. 


\section{The Agriculture Scene}

Agricultural scientists have a few additional advantages and disadvantages when it comes to public awareness of science. As more and more Canadians live in urban settings, they become less and less aware of agriculture. This means that fewer and fewer people appreciate the necessity of or benefits offered by agricultural operations, let alone the problems faced by this essential component of our society.

The isolation is not just confined to members of the general public. Many Canadian scientists are urban creatures whose work is more basic. The structure of educational and research institutions has usually offered them little contact with their agricultural counterparts. This isolation translates to little communication and a lack of appreciation. I can use myself as an example.

As someone who was raised and has lived and worked in cities, I have had little exposure to agricultural science. Furthermore, my work in ecology and behaviour often gave me reason to be critical of the impact that agriculture can have on the environment. Ibegan to change in 1990 when I visited a friend on a ranch in Zimbabwe. He is a wildlife biologist now working as a ranch manager. One evening, as we listened to jackals calling, my friend noted that it was time to shoot a few more of these pests. I could see no reason to think this way and accused him of having lost contact with his wildlife roots. The next day he showed me why he views jackals as a nuisance. A cow having a difficult birth had been attacked by jackals that had eaten the calf's tongue and part of his face and seriously injured the cow's vulva. Both cow and calf had to be put down. This is a recurring problem in beef cattle operations where there are high jackal populations.

Having seen the effect of the jackals, I could appreciate how easy it is to ignore the problems faced by other workers and condemn what you perceive to be an unenlightened view of the world.

Gardening is a big plus for agricultural scientists. This pastime offers millions of Canadians a glimpse of the problems and contributions of agriculture and agricultural science. Gardening gives people a first-hand introduction to the business of accurate measurements (perhaps of fertilizer or pesticide); to some basic genetics, and to the problems facing those whose business is growing plants for decoration or consumption.

\section{Action}

It is tempting to think that scientists should and could rely on the media to assist them in their efforts to change the public's view of science. We are fortunate in having excellent science reporters who address science issues and discoveries on print, on radio, and on television. This media coverage can 
have great impact on the public, for example, the hype surrounding the recent Canadian foray into space, but I do not think that the press will substantially change the public's view of science.

Scientists themselves should take a more active role in changing the future of their disciplines. Several activities come to mind. First, scientists should get out and communicate with non-scientists. This can be done in many settings, but one of the best is local school rooms where elementary or secondary school students are learning about science. Visit a classroom, talk about your work-what you are doing and why. You will leave the class with a strong impression. The students will share this with their parents and your time investment can pay dividends in the long run.

If you have ideas about science projects that could be operated in elementary or secondary school classrooms, share them. You can speak about them to teachers at local schools or, if you want broader coverage, there is another opportunity: Recently, Science Culture Canada awarded the Canadian Federation of Biological Societies and the World Wildlife Fund Canada support for a project designed to bring science projects to the classroom. We are looking for projects that address broad issues of the environment: conservation and wildlife, conservation and genetics, conservation and toxicology. I will be co-ordinating the work and would appreciate receiving your ideas. We will employ an experienced teacher to work with scientists to develop, polish, and test the exercises with a goal to producing a final product by July, 1993.

Scientists whose responsibility includes broader management roles can ensure that those working under them receive credit for trying to increase public awareness of science. These activities are usually taken for granted, but this could change quickly if Chairs and Deans in universities and Directors in government labs overtly recognized and encouraged these vital contributions.

If you think that scientists do communicate about basic research, consider the situation in universities. For those of us who work there, it is sobering to realize that many graduates emerge with no appreciation of the role that research plays in universities. We can easily set this right by making sure that undergraduate students are repeatedly exposed to research activity. At one level, they should know about the jobs generated by research, and at another level, realize how much of their learning experience is enriched by the research done by graduate students and professors. If everyone pitched in on this front, we could quickly dispel the notion that university faculty enjoy four months of paid vacation every year. 
In closing, I find reasons to be optimistic about the future of basic and applied research in Canada. If scientists choose to share the excitement and enthusiasm generated by the quest for answers, they could change the public awareness and appreciation of science. The ball is in our court! 